\title{
Multicystic Facial Nerve Neurinoma: A Case Report
}

\author{
Veysel Antar ${ }^{\mathrm{a}}$, Oguz Baran ${ }^{\mathrm{a}, \mathrm{c}}$, Salim Katar ${ }^{\mathrm{a}}$, Kadir Kotil ${ }^{\mathrm{b}}$
}

\begin{abstract}
Facial nerve neurinoma (FNN) can present as masses in the internal auditory canal or cerebellopontine angle and may mimic an acoustic neurinoma. A 44-year-old male presented with a huge facial schwannoma extending into both the middle cranial fossa and the cerebellopontine angle but without manifesting facial nerve palsy. Neurological examination on admission revealed no deficits except for speech disturbance. Magnetic resonance images (MRIs) showed a multicystic tumor extending into the middle cranial fossa and the cerebellopontine angle, with destruction of the petrous bone. The tumor was totally grossly removed. Histological examination identified schwannoma. Facial nerve palsy is one of the most typical symptoms in patients with facial schwannoma, but is not always manifested even if the tumor extends into both the middle cranial fossa and the cerebellopontine angle. They must be differentiated both clinically and radiologically from acoustic neurinomas.
\end{abstract}

Keywords: Facial nerve neurinoma; Cerebellopontine angle; Facial schwannoma

\section{Introduction}

Facial nerve neurinomas (FNNs) are relatively uncommon, and slow growing lesions that can involve any nerve segment [1]. FNN extending into both the temporal fossa and the cerebellopontine angle occurs in only $3 \%$ of all cases, and almost always present with facial nerve palsy and hearing loss persisting for 4 months to 17 years [2]. FNN has various manifestations, including facial palsy, hearing loss, vestibular weakness,

Manuscript accepted for publication July 01, 2015

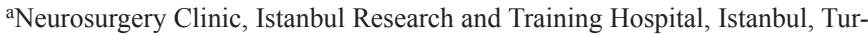
key

${ }^{b}$ Department of Neurosurgery, Istanbul Arel University Medical Faculty, Istanbul, Turkey

${ }^{\mathrm{c} C}$ Corresponding Author: Oguz Baran, Istanbul Egitim ve Arastirma Hastanesi, Beyin ve Sinir Cerrahisi Klinigi, Kasap Ilyas Mah, Org. Nafiz Gurman Cad, Samatya, Istanbul 34098, Turkey. Email: oguzbaran@gmail.com

doi: http://dx.doi.org/10.14740/jmc2241w a palpable parotid mass and even no symptom depending on its size and site $[1,3]$. Unfortunately, facial nerve is very resistant to compression, and often facial nerve paralysis or a facial weakness is not present, for this a normal facial nerve function occurs in $27.3 \%$ of all patients with FNNs [4]. Preoperative diagnosis of FNN manifesting no facial nerve paresis is quite difficult. We present a case of FNN who presented with features suggestive of middle temporal fossa multicystic tumor but without manifesting facial nerve palsy.

\section{Case Report}

A 44-year-old man was admitted to a local clinic, because of left tinnitus with decreased hearing in the left ear for 7 years. On admission, physical examination showed no facial palsy, tinnitus and sensorineural hearing loss on the left side. Cranial magnetic resonance images (MRIs) demonstrated a multicystic mass sized $5.1 \times 4.0 \times 4.5 \mathrm{~cm}$ at the left middle cranial fossa and the meatus acusticus internus (Fig. 1A, B). Left internal auditory canal diameter was enlarged, and the mass extended from the internal auditory porus to the middle fossa, transecting the petrous bone. Thus, the patient was diagnosed as having neurinoma with extending into the temporal fossa. But MRI arrived at a diagnosis of FNN rather than acoustic neurinoma, since the petrous bone was eroded along the facial canal toward the geniculate ganglion. The surgery was made by left infratemporal fossa approaches with microneurosurgery. During the operation, the surgeons found that the tympanic cavity and sinuses were filled with reddish-gray neoplasm, which eroded the mastoid and petrous bone and invaded into the cranial cavity. Then, xanchromatic fluid was aspirated from the cystic mass in the middle cranial fossa. Tumor was totally extirpated under the facial nerve monitoring. Facial nerve was preserved. The diagnosis of neurinoma was confirmed by postoperative histological examination. Histologic examination of the specimen showed schwannoma which was included hypercelularity, xsantomatic histiocytic reaction, hyalinization and compact elongated cells $(\times 100$, H\&E). Postoperatively, on neurological examination, facial weakness with House-Brackmann Grade II was noted. After 8 months, facial weakness was resolved spontaneously. The patient showed a complete hearing loss in the left ear, but the facial sensation and visual acuity were normal. The patient was followed up 


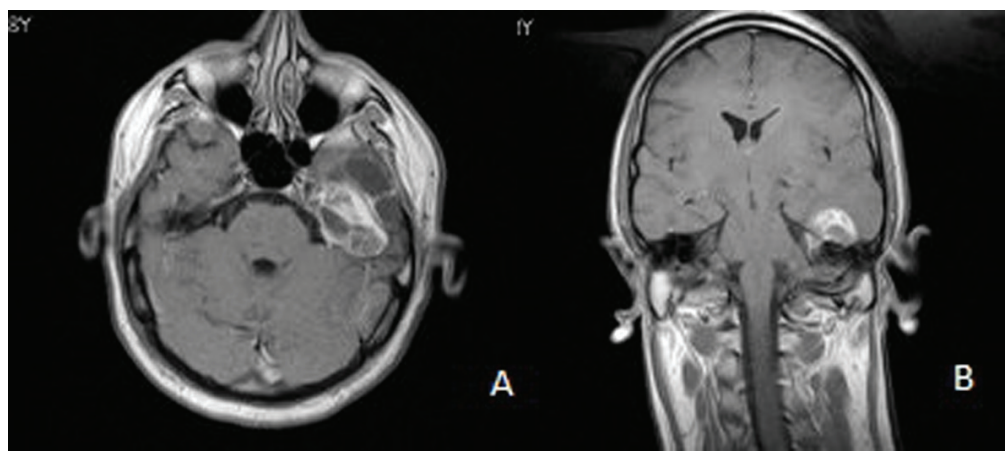

Figure 1. MRI scans showed an enhancing cerebellopontine angle fusiform tumor: (A) with considerable internal auditory canal and middle cranial fossa multicystic tumor; (B) the multicystic tumor occupied the petrous bone and extended anteriorly and upwardly into the left middle cranial fossa.

for 2 years, and no enlargement of internal acoustic segment tumor recurrence was detected (Fig. 2).

\section{Discussion}

FNNs involving the middle cranial fossa are especially rare [3]. It has been noted that there is a predilection for FNN to arise from the area of geniculate ganglion, which might be attributable to the major structural reorganization occurring between the labyrinthine and tympanic segments of the facial nerve [1]. FNN is a rare cause of facial palsy accounting for less than $5 \%$

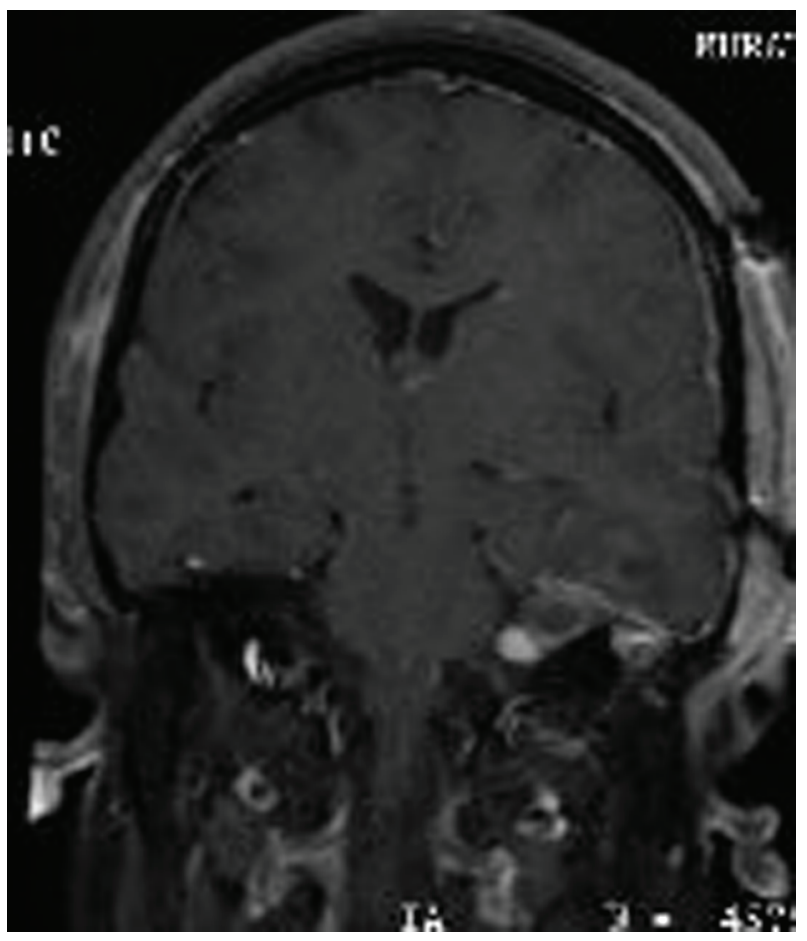

Figure 2. Postoperative coronal T1-weighted MRI with gadolinium enhancement. The multicystic tumor was resected by the left side middle cranial fossa craniotomy (infratemporal approach). But internal acoustic canal tumor was not removed. It has been observed for 2 years. of cases $[4,5]$. Insidious onset of facial palsy, recurrent facial palsy and persistent facial palsy was after 12 weeks. This case has not paresis of his face. Enlargement of the auditory canal and erosion of the petrous bone along the facial nerve proximal to the geniculate ganglion might have prevented compression of the facial nerve and consequent impairment of its function. As mentioned, the absence of facial nerve dysfunction is probably attributable to the bony erosion, which allowed adequate space for the facial nerve.

MRI is preferred to detect an FNN, even in a small size, and to clearly delineate the location and the extent of the tumors, which is particularly important for the resection of the tumor and the preservation of the facial nerve. MRI of an FNN shows mildly hypo- or hyper-intense to grey matter on T1weighted images and hyper-intense on T2-wighted images, which can be markedly enhanced by using gadolinium. Cystic change commonly shown in other neurinomas is occasionally seen in large facial neurinomas [6, 7]. The initial accurate diagnosis of FNNs involving the middle cranial fossa is challenging. Differential diagnosis of dumbbell-shaped tumor located in the middle cranial fossa and cerebellopontine angle includes cholesteatoma, trigeminal schwannoma (TS), cavernous hemangioma and acoustic schwannoma (AS).

In this case, enlargement of the internal auditory canal and erosion of the middle portion of the petrous bone by bone CT, postoperatively, lead to the preoperative diagnosis of facial schwannoma despite the normal facial nerve function.

\section{Grant Support}

There is no grant.

\section{Conflict of Interest}

There is no conflict of interest.

\section{References}

1. Chen JM, Moll C, Wichmann W, Kurrer MO, Fisch U. 
Magnetic resonance imaging and intraoperative frozen sections in intratemporal facial schwannomas. Am J Otol. 1995;16(1):68-74.

2. McRackan TR, Rivas A, Wanna GB, Yoo MJ, Bennett ML, Dietrich MS, Glasscock ME, et al. Facial nerve outcomes in facial nerve schwannomas. Otol Neurotol. 2012;33(1):78-82.

3. Symon L, Cheesman AD, Kawauchi M, Bordi L. Neuromas of the facial nerve: a report of 12 cases. Br J Neurosurg. 1993;7(1):13-22.

4. Clark WC, Acker JD, Robertson JH, Gardner G. High res- olution computed tomographic scanning in the detection of intratemporal neuromas of the facial nerve. Neurosurgery. 1983;13(6):672-675.

5. Vrabec JT, Guinto F, Jr., Nauta HJ. Recurrent facial neuromas. Am J Otol. 1998;19(1):99-103.

6. Parnes LS, Lee DH, Peerless SJ. Magnetic resonance imaging of facial nerve neuromas. Laryngoscope. 1991;101(1 Pt 1):31-35.

7. Nadeau DP, Sataloff RT. Fascicle preservation surgery for facial nerve neuromas involving the posterior cranial fossa. Otol Neurotol. 2003;24(2):317-325. 